Rev. Elev. Méd. vét. Pays trop., 1972, 25 (1): 109-118

\title{
Une amélioration spectaculaire en production laitière dans la vallée du rio Cauca en Colombie Méthodes et résultats
}

\author{
par P. PONSARDIN $\left({ }^{*}\right)$
}

\begin{abstract}
RESUME
Dans cette très fertile vallée d'altitude $(900$ à $1000 \mathrm{~m})$ à climat tropical chaud et humide ( ${ }^{\circ}$ de latitude nord), un groupe d'experts colombiens et français agissant à la fois sur le pâturage, l'animal et l'éleveur, a recherché par l'emplo1 raisonné de méthodes classiques adaptées au milieu, la modification des qualités de l'exploitant, dans sa gestion et sa technicité.

Cet article analyse les intéressants résultats obtenus sur 8 exploitations pilotes comportant au total 650 vaches. La production laitière y est passée en 2 ans de $4000 \mathrm{~kg}$ de lait $/ \mathrm{ha} /$ an à $5900 \mathrm{~kg} / \mathrm{ha} / \mathrm{an}$, soit une augmentation de production de 47 p. 100 environ avec une très forte amélioration de la rentabilité.

L'objectif du programme prévoit l'assistance à 200 exploitations couvrant 25000 ha avec, sur les bonnes fermes laitières, une production de $8000 \mathrm{~kg}$ de lait/ha/an (déjà obtenue sur certaines exploitations).

Le pays manque de lait, d'où l'intérêt économique et social de ce programme.
\end{abstract}

\section{INTRODUCTION}

\section{LA MISSION TECHNIQUE FRANÇAISE DaNS La VALleE du CAUCa : REGION DE CALI (COLOMBIE)}

Dans le cadre de la Coopération technique bilatérale, le Gouvernement Français a mis, à la fin de l'année 1967, trois experts de SCETInternational à la disposition de la C.V.C. (Corporacion Del Valle del Cauca), société para-publique d'aménagement régional. Cet organisme d'intérêt public a pour vocation le développement économique intégral de la région placée sous sa juridiction, et comporte entre autres un département agricole au sein duquel la Mission Française a organisé, animé et déve-

(*) Docteur vétérinaire, zootechnicien. Société centrale pour l'Equipement du Territoire-International, 5-7, rue Bellini, 92-Puteaux. Filiale technique de la Caisse des Dépôts et Consignations. loppé un groupe polyvalent d'administration de fermes.

A noter que cette région est de loin la première région agricole de Colombie assurant selon les dernières statistiques 33 p. 100 de la production agricole du pays et 25 p. $100 \mathrm{du}$ total de ses exportations.

Depuis le début de l'année 1969, la Mission française comprend deux experts : 1 agro-économiste et 1 vétérinaire-zootechnicien travaillant en étroite collaboration avec les cadres colombiens représentés à l'heure actuelle par 10 ingénieurs agronomes, 3 vétérinaires-zootechniciens et 2 économistes agricoles. $\mathrm{Ce}$ groupe, dirigé par un ingénieur agronome, a été constitué pour apporter une assistance technique aussi complète que possible aux exploitations agricoles et aux fermes d'élevage de la région. 


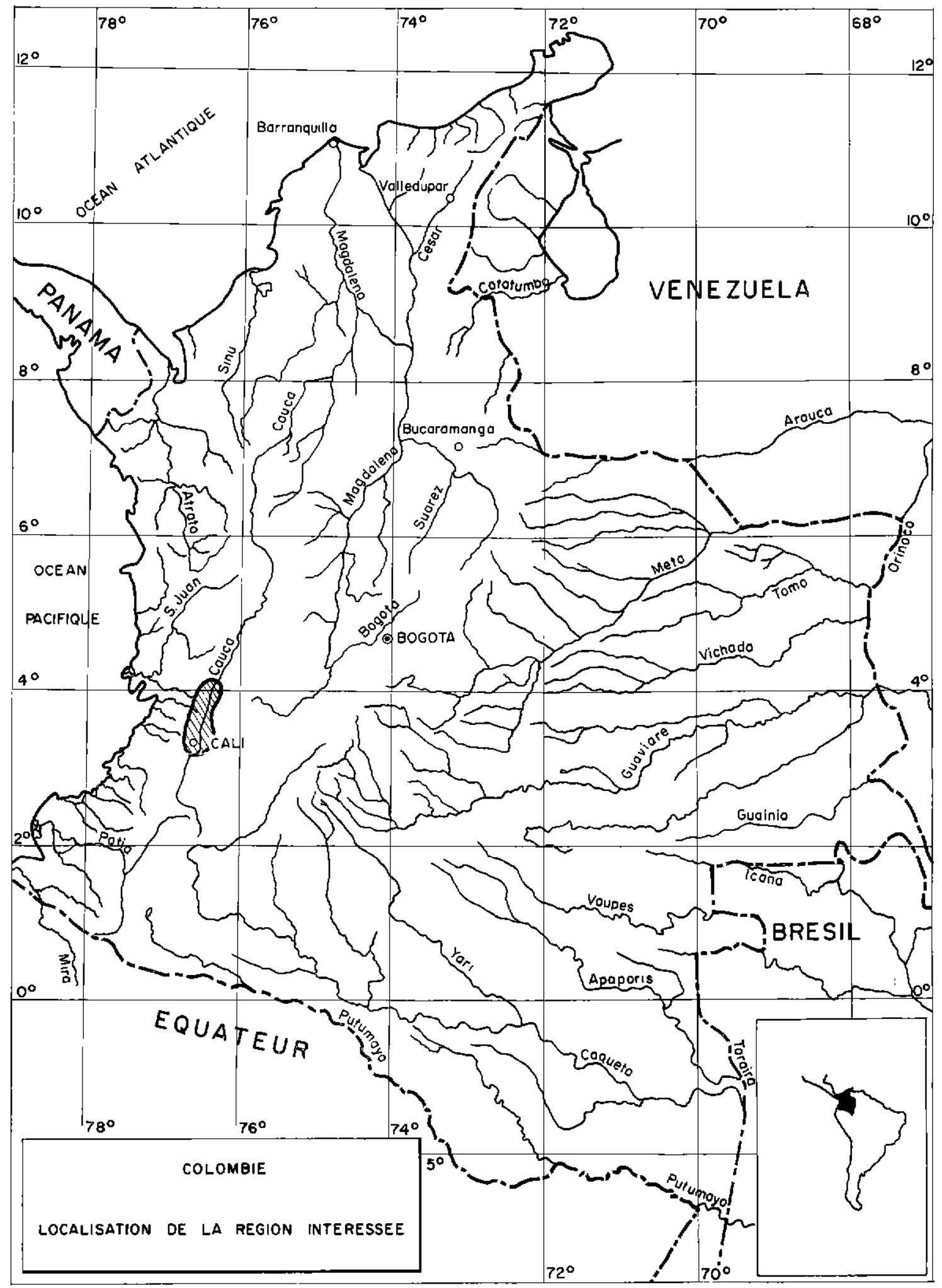


Son objectif est de transformer les exploitants agricoles en véritables chefs d'entreprise, en améliorant leurs qualités d'administrateur, ainsi que leurs connaissances techniques et économiques.

La méthode de travail retenue est celle de la gestion des exploitations agricoles développée en France par le Professeur CHOMBARD de LAUWE (analyses technico-économiques d'exploitations, fixation d'objectifs, études de groupes d'exploitation, plans de réorganisation d'exploitations, visites de contrôle).

Cette méthode de gestion fut appliquée avec plus de "densité ", en l'adaptant au milieu (fréquence des contrôles, mesures périodiques des résultats) et fut plus "étoffée " sur le plan technique. Le conseil de gestion fut plus " intégral ", plus détaillé. Par exemple en élevage, les conseils ont porté tant sur le pâturage (choix de variétés, fertilisation, drainage, irrigation, division en parcelles, programme de rotation par groupe d'animaux) que sur l'animal (programmes d'alimentation, conseils de sélection, élevage des veaux, hygiène de la traite, contrôle sanitaire, introduction de registres d'étable pour une meilleure conduite du troupeau).

Cette infrastructure apparemment coûteuse a priori a porté ses fruits, comme nous le verrons plus loin.

La méthode de travail du groupe, ainsi que sa "philosophie " sont appréciées de l'organisation de tutelle qui a appliqué depuis 2 ans la nouvelle politique d'administration par fixation d'objectifs et auto-contrôle du personnel.

Le groupe chargé de fixer les objectifs et de contrôler périodiquement les résultats était parfaitement adapté à cette nouvelle politique.

Le service, gratuit au début pour les exploitants, est devenu payant depuis plus dun an. Cette mesure a permis la sélection des bénéficiaires et a été salutaire. Le but fixé est d'assurer l'autofinancement du groupe d'ici 4 ans, la subvention de l'organisation diminuant de $25 \mathrm{p}$. 100 chaque année.

Pour la fin de l'année 1971, Je groupe s'est fixé comme objectif l'assistance à 200 exploitations, d'élevage ou mixtes, couvrant au total 25.000 ha environ.

Nous pouvons considérer cette expérience comme très encourageante et unique en son genre, peut-être en pays tropical.

\section{CONDITIONS NATURELLES DE LA REGION - ASPECTS DE LA PRODUCTION LAITIERE ET ACTIONS ENTREPRISES}

\subsection{Conditions naturelles de la Vallée}

La vallée du Cauca s'étire sur $300 \mathrm{~km}$ environ selon la direction sud-ouest nord-est, sa largeur variant de 10 à $40 \mathrm{~km}$. Elle a un peu la forme d'un violon vu de profil. Située dans la zone intertropicale mais très près de la ligne équatoriale $\left(4^{\circ}\right.$ de latitude nord, $76^{\circ}$ de longitude ouest en son centre) l'altitude est comprise entre 900 et $1.000 \mathrm{~m}$ (Cali); chaud (température variant entre 20 et $35^{\circ} \mathrm{C}$, moyenne de $25^{\circ} \mathrm{C}$ ) avec des variations mensuelles et nycthémérales plutôt faibles. Par contre l'humidité relative est assez élevée (moyenne 70 p. 100).

La pluviométrie est élevée (variant de 1.000 à $2.000 \mathrm{~mm}$ d'un point à un autre) et assez bien répartie. Cependant on distingue annuellement 2 saisons des pluies alternant avec 2 saisons plus sèches. De toute façon, l'irrigation d'appoint nécessaire est presque toujours possible.

La vallée géographique du Rio Cauca s’étend sur 350.000 ha et possède les sols les plus fertiles de Colombie dont environ 200.000 ha de sols très riches d'origine alluviale. Une partie de ces sols est cependant affectée actuellement d'inondations temporaires (60.000 ha).

\subsection{Aspects de la production laitière et actions entreprises}

\subsubsection{Les exploitations et les éleveurs}

-- La taille des exploitations laitières est très variable : elle va de 15 à 150 ha mais la grande majorité des fermes comprises actuellement dans le programme a une superficie qui s'étage entre 20 et 100 ha et est donc de taille moyenne et grande. A ce sujet, 100 ha peuvent être considérés actuellement pour le pays comme la surface maximale à consacrer à une exploitation laitière intensive (les facteurs limitants étant le capital d'exploitation nécessaire et également la capacité administrative des propriétaires ou administrateurs).

- Le mode de faire-valoir : direct en général 
mais très souvent par personne interposée, le propriétaire de l'exploitation a bien souvent une autre occupation et délègue en partie ses fonctions à un administrateur ou à un contremaître d'élevage qui ont un niveau de formation et une valeur professionnelle très variables.

- Les éleveurs : sont bien souvent d'un niveau intellectuel supérieur (médecins, avocats, ingénieurs, industriels, hommes d'affaires) mais pèchent à l'origine par un manque de formation technique et administrative si nécessaire à l'élevage moderne. Toutefois, ils sont bien préparés au calcul économique, en sentant rapidement l'impérieuse nécessité :

A) Ils sont devenus propriétaires soit en héritant, soit en achetant par " hobby " pour investir leurs capitaux et retourner un peu à la terre. On peut les convaincre de transformer, à l'aide d'un programme soutenu si besoin par le crédit, leurs exploitations en entreprises d'un intérêt économique certain et même parfois très intéressant.

B) La sélection des éleveurs se déroule ainsi : le programme n'est pas imposé et bien qu'il ne soit pas gratuit, tous les candidats ne peuvent y prétendre. A la suite d'une entrevue au bureau avec le propriétaire demandeur et la visite très détaillée de l'exploitation (toujours en présence du propriétaire), on se rend compte si le candidat peut entrer dans le programme et, peu après, on le lui notifie en Iui réclamant un droit d'entrée réduit.

Ensuite, il faut aider ces éleveurs choisis à administrer leurs biens, élaborer d'un commun accord un programme de travail par étape, fixer les inversions nécessaires, les aider à obtenir un crédit le cas échéant. Il faut souvent les former techniquement, eux et leurs aides.

Il est nécessaire de résoudre, soit directement, soit indirectement, les problèmes techniques de l'exploitation: amélioration du pâturage, amélioration du bétail (intérêt des registres de production et de reproduction, début de sélection). Si la comptabilité existante permet de faire la première analyse économique, celle-ci est entreprise et va permettre de déterminer avec précision les points faibles de l'exploitation. Sinon, on aide l'éleveur à installer et à tirer partie d'une comptabilité agricole simple et fonctionnelle mise au point par le groupe. L'éleveur acquiert peu à peu avec l'apparition des premiers résultats (souvent spectaculaires) une grande confiance à la fois dans le technicien qui l'aide et dans le programme et peu à peu, lui-même s'améliore, se transformant en un véritable chef d'entreprise.

C'est bien là le but, la finalité de notre forme de coopération (la formation intégrée des ingénieurs et des exploitants " au ras du sol ", dans le milieu même et ses difficultés).

On a prétendu que cette politique de coopération était limitée dans l'espace, coûteuse et par là-même se traduisait par une productivité relativement faible. Mais dans le cas présent, les tableaux des résultats qui suivent et que nous devons publier prouvent que notre coopération a porté ses fruits.

Par l'emploi de méthodes et de techniques bien adaptées au milieu et aux hommes on a pu, en peu de temps, améliorer de façon spectaculaire l'économie de l'élevage dans une région privilégiée (au potentiel élevé) mais sousdéveloppée, donc par là-même bien choisie.

\subsubsection{Le pâturage}

Le pâturage naturel de la Vallée, à base de " trenza" (Paspalum notatum) est très peu productif, seules les zones humides ou plutôt à niveau phréatique élevé sont recouvertes d'une graminée naturelle de valeur et très productive: l'herbe de Para (Panicum purpurescens) ( $\left.{ }^{(}\right)$. L'herbe de Guinée (Panicum maximum) croît également spontanément sur les bonnes terres, de même que Cynodon dactylon sur les terres salées.

Mais le Pangola (Digitaria decumbens) est la base du pâturage amélioré et se répand de plus en plus avec l'intensification des exploitations. Quand il est bien exploité, il peut durer de nombreuses années (8 - 10 ans). On doit surtout éviter le surpâturage (récupération très lente et développement d'adventices). Pour produire au maximum, le Pangola doit évidemment être bien exploité : division des herbages, rotation des parcelles, repos suffisant de chaque parcelle, irrigation d'appoint pour les jours secs, fertilisation suffisante à base de scorie 2 fois par an, et d'urée après chaque passage du bétail ( $800 \mathrm{~kg}$ d'urée par ha et par an en 10 applications environ, chaque 30 à 40 jours).

De cette manière on a pu obtenir des charges à l'ha très élevées et cela durant toute l'année.

(1) Brachiaria mutica des agrostologues fránçais. 
Les objectifs fixés pour les bonnes terres consacrées à l'élevage laitier intensif sont :

- 4 UGB (unité gros bétail) (dont 2,7 vaches environ) par ha de S.F. (surface fourragère);

- plus de $8.000 \mathrm{~kg}$ de lait/ha/an.

Ces objectifs sont déjà dépassés dans certaines exploitations d'élite.

Si le pâturage bien exploité a un rendement très élevé, soutenu, et autorise une très forte charge à l'hectare, dans ce milieu tropical, la production moyenne des vaches est limitée par la chaleur du jour (les animaux souffrent et dépensent de l'énergie pour maintenir leur température interne). Ainsi, la production par hectare compense très largement la limitation de la production individuelle.

Peu de légumineuses résistent à une telle intensification. Les petites, du genre Desmodium, sont les seules qui subsistent.

Le pâturage est très souvent complémenté durant les heures chaudes de la journée par du fourrage de coupe, distribué à l'auge en vert et de la meilleure qualité possible. Le fourrage le plus utilisé et le plus recommandable à la fois par ses rendements très élevés (plus de 300 tonnes/ha/an de matière verte) et sa valeur nutritive au stade d'exploitation est l'( Elefant grass " (Pennisetum purpureum); les légumineuses de coupe ne sont pratiquement pas utilisées actuellement; les essais de luzerne ont donné généralement de mauvais résultats à cause du niveau souvent trop élevé de la nappe phréatique. La ramie $\left({ }^{1}\right)$ a été essayée à grande échelle comme fourrage de coupe mais ses rendements annuels sont faibles et, en fin de compte, la production annuelle à l'ha en U.F. (unité fourragères) et de protéines digestibles est beaucoup plus élevée avec l'a Elefant grass".

\subsubsection{Les animaux}

Les races utilisées sont essentiellement au nombre de 2: la "pardo suiza" (Braun Schwitz américaine) et surtout la Holstein (80 p. 100 des effectifs environ). Les anciennes races locales ou les femelles métissées de zébu qui étaient traites (avec le veau) une fois par jour et élevées de façon très extensive, disparaissent de plus en plus avec l'intensification. Par contre elles subsistent dans les régions plus

(1) Urticacée : Boehmeria nivea. pauvres de Colombie et fournissent toujours la grande majorité du lait produit dans le pays.

Le niveau de sélection demeure encore généralement très bas et très variable d'une ferme à l'autre, il dépend avant tout du niveau technique et administratif de l'éleveur lui-même.

On a pensé longtemps et on continue à penser trop souvent que la seule amélioration par le mâle (en monte directe ou par insémination artificielle) était suffisante au progrès génétique et dispensait de la sélection des femelles laitières. Grave faute que les éleveurs convaincus réparent de plus en plus vite en installant et en utilisant les registres d'étable qui permettent à la fois la sélection des vaches à l'aide des critères de reproduction et de production laitière (contrôle laitier) et le choix rationnel des jeunes animaux d'élevage.

\subsubsection{La conduite du troupeau}

La mauvaise conduite du troupeau est encore un des facteurs limitants essentiels de la production, on voit trop souvent les taureaux en monte libre et l'absence totale de contrôle.

La séparation du taureau, le diagnostic périodique de gestation, l'installation du calendrier d'étable et des fiches individuelles après identification des animaux, avec colliers et plaques numérotées, permettent en peu de temps des progrès spectaculaires, surtout si on améliore en même temps les conditions alimentaires.

Les mesures élémentaires consistant à faire saillir les vaches durant le $3^{*}$ mois qui suit le vêlage et à les tarir 2 mois avant la mise-bas étaient bien souvent ignorées, ou mal appliquées.

La répartition rationnelle en lots d'animaux était également très négligée: très souvent les jeunes génisses d'élevage, mélangées aux animaux adultes, étaient la proie des parasites très prolifiques dans ce milieu chaud et humide. Depuis, les animaux ont été séparés en lots, chaque lot bénéficiant de ses propres parcelles exploitées en rotation.

L'éleveur allait à l'aventure et ignorait généralement le nombre souhaitable d'animaux par catégorie et en particulier le nombre de velles à élever chaque année. Le plan de réorganisation de l'exploitation comporte dans ce sens une analyse détaillée des catégories d'animaux correspondant à l'effectif prévu. 
Vaches et génisses de plus de 6 mois vivent toute l'année dehors. Des arbres d'ombrage doivent être prévus à cet effet dans les herbages. Les vaches ne rentrent à l'étable (conçue très économiquement) que pour la traite et la consommation à l'auge du fourrage de coupe.

L'élevage des velles a lui aussi été nettement amélioré; grâce à l'isolement en cages individuelles des jeunes animaux, sevrage précoce et rationnel, meilleure répartition du lait et des concentrés, on a obtenu une amélioration spectaculaire des résultats économiques et zootechniques.

\subsubsection{L'alimentation complémentaire du pâturage}

- L'aliment concentré : il était généralement mal utilisé. On l'employait trop ou trop peu, presque jamais en fonction de la production des femelles et pour cause puisqu'on ignorait la production exacte de celles-ci.

Aussi utilisait-on mal le pâturage, source d'unités fourragères à meilleur marché, surtout dans ce pays, et essayait-on de compenser la faible production d'animaux mal sélectionnés par l'apport fait au hasard de concentré. Ces erreurs zootechniques et d'alimentation conduisaient à de mauvais résultats économiques : l'incidence sur la productivité laitière était nulle mais le coût de production du lait était inutilement accru.

D'autre part, les concentrés commerciaux sont souvent de faible valeur nutritive, surtout énergétique (défaut de contrôle officiel). On a dû vulgariser la préparation des concentrés à la ferme, les matières premières (céréales, mélasse et tourteaux) étant produites dans la région.

Soulignons également le rôle joué par le fourrage de coupe (Pennisetum purpureum), distribué à l'auge, hâché et imbibé d'une solution de mélasse de canne pour augmenter la palatibilité. Les animaux adultes arrivent à consommer jusqu'à $30 \mathrm{~kg}$ par tête de ce fourrage: la quantité consommée variant bien entendu avec l'animal, la valeur de ce fourrage, la valeur du pâturage surtout et bien entendu le nombre d'heures passées à l'auge.

Certains éleveurs ont abandonné la distribution de sous-produits industriels, de très faible valeur nutritive mais appétés qui limi- taient la consommation de bonnes graminées par les bovins (coques de soja et même cacao).

\section{- Le complément minéral :}

On ignorait son importance. On ne pensait qu'au sel. On oubliait l'importance des autres minéraux surtout du phosphore, en général déficient dans les fourrages tropicaux, surtout pour des animaux améliorés aux besoins élevés. Et là encore les compléments minéraux du commerce étaient nettement insuffisants. Dans ce sens, les compléments minéraux se préparent maintenant bien souvent à la ferme, à base de phosphate bicalcique alimentaire importé d'Europe.

La complémentation s'effectue à l'auge, en mélange avec le fourrage de coupe très appété. Par contre le sel marin est toujours placé à la libre disposition des animaux au pâturage, à l'abri des intempéries.

\subsubsection{Amélioration du contrôle sanitaire}

- Les affections virales et microbiennes :

Ces maladies, dont la plus grave est la fièvre aphteuse, sont bien contrôlées en général dans la région, sauf la brucellose qui sévit à l'état enzootique. Ici encore les mesures ont été prises à l'échelle de chaque ferme assistée. Les animaux à réaction sérologique positive sont peu à peu éliminés (aucune aide de l'Etat dans ce domaine) et toutes les génisses d'élevage sont vaccinées à l'âge de 6 mois avec le vaccin $B_{19}$.

Un grand travail d'information a été fait également auprès des éleveurs, leur indiquant toutes les précautions nécessaires avant tout achat de bétail (maladies vénériennes et autres).

La tuberculose bovine est absente de la région. Très peu de cas de rage chez les bovins. Les mammites sont en général bien contrôlées.

- Le parasitisme, tant interne qu'externe, est le problème sanitaire majeur; cependant les moyens de contrôle ne manquent pas et sont excellents.

Aussi, les pertes peuvent être incriminées à la négligence ou à l'insuffisance de gestion de nombreux éleveurs. Il existe actuellement d'excellents parasiticides. On sait d'autre part que la rotation rationnelle des jeunes animaux dans des parcelles qui leur sont propres aide parfaitement au contrôle des parasites (en association avec la destruction des broussailles). 
Les exploitations possèdent bien souvent un bain détiqueur ou une installation par aspersion (Cooper) mais les erreurs techniques sont nombreuses : la périodicité des traitements n'est pas respectée et on utilise trop longtemps le même produit et les tiques deviennent chimio résistantes. On peut dire que dans la région 80 p. 100 des cas de mortalité des animaux (mortalité relativement très faible) sont dus à des maladies transmises par les tiques (Piroplasmose, babesiellose et anaplasmose). Ces affections frappent surtout les animaux récemment achetés, non prémunis contre les souches locales de parasites.

La trypanosomose transmise par des insectes piqueurs (taons et stomoxes) existe surtout dans le sud de la Colombie mais son incidence n'est pas comparable à celle de son homologue africain.

En général on peut dire que. dans cette région, la pathologie n'est pas un facteur limitant de l'élevage. Le contrôle sanitaire ne pose aucun problème particulier. $\mathrm{Ce}$ pays est indemne des grands fléaux de l'élevage africain tels que la peste bovine et la péripneumonie contagieuse.

\subsubsection{Le marché du lait - Les dérivés laitiers}

Ici il n'y a pas lieu de craindre la surproduction Iaitière; le pays manque de lait. La consommation actuelle a été évaluée à 0.16 litres per capita par jour et encore est-elle très mal répartie (dans les catégories de faibles revenus, elle atteindrait seulement 0,07 litre ...).

Le " mouillage " de cet aliment est d'autre part généralisé et ceci, bien entendu, dans les quartiers populaires des villes.

Par ailleurs, dans une grande ville comme Cali (1 million d'habitants), 40 p. 100 seulement du lait vendu est pasteurisé. Or la pasteurisation devrait être généralisée et obligatoire dans ce milieu tropical où, de nlus. le lait cru est toujours altéré ou l'objet de fraude avant sa consommation. Le lait n'est généralement pas refroidi à la ferme. l'investissement supplémentaire nécessaire n'étant pas compensé actuellement par un prix d'achat supérieur.

Quoi qu'il en soit, le lait est vendu à un prix rémunérateur à la production : le prix d'achat du litre variant entre 0,11 et 0,13 US $\$$ (selon la distance par rapport à la ville). Il "paie" actuellement beaucoup plus que la viande bovine en élevage intensif : le prix producteur de la viande représente l'équivalent de 0,35 US $\$$ le $\mathrm{kg}$ vif sur pied (soit environ $0,70 \mathrm{US} \$$ le $\mathrm{kg}$ de viande nette sur pied, soit 6 fois plus seulement que le litre de lait). A charge égale et malgré des coûts, en particulier de main-d'œuvre, plus élevés, dans ces terres riches, le lait est beaucoup plus rentable que la viande ainsi que l'ont prouvé les études technico-économiques. Il est vraisemblable que cette tendance se maintiendra et même s'amplifiera dans les années à venir : la demande du lait ne faisant que s'accroitre et la viande de bovin pouvant être remplacée en partie par d'autres viandes à production plus rapide et facilement industrialisables comme le porc et le poulet. Ces derniers sont actuellement hors de prix, ce sont de véritables viandes de luxe (le contraire de nos pays d'Europe; ceci est dû surtout à la forte incidence du coût alimentaire : maïs à 95 US $\$$, sorgho 85 US $\$$, tourteau de soja 130 US $\$$ la tonne ...). Quant au poisson, il est encore trop peu consommé (problèmes d'équipements et d'éducation du consommateur).

On fabrique très peu de dérivés du lait, le plus connu est le fromage frais, trop souvent fabriqué de façon artisanale dans des conditions d'hygiène plutôt douteuses.

Les glaces se consomment très bien; par contre on fabrique très peu de yogourt, vendu toujours très cher. Les autres fromages et le beurre viennent des régions froides (Bogota); le marché de ces produits est limité actuellement surtout par leur prix très élevé par rapport au pouvoir d'achat de la grande majorité des consommateurs potentiels.

\section{RESULTATS OBTENUS ET CONCLUSIONS}

Après 3 années, on a pu obtenir des résultats positifs spectaculaires qui assurent au programme un avenir prometteur. En effet, ces résultats sont publiés et divulgués, lors de réunions d'éleveurs, tenues dans la ferme même d'un adhérent. Le progrès fait ainsi tache d'huile et les objectifs que s'est fixé le programme doivent être atteints.

Les analyses économiques d'exploitations élaborées depuis l'année 1968 ont pu être aisément synthétisées et ont conduit aux tableau I et II de résultats ci-après. 
TABLEAU $\mathrm{N}^{\circ} \mathrm{I}$

Evolution de quelques exploitations du programme

(Résultats réels d'analyses technico-êconomiques)

\begin{tabular}{|c|c|c|c|c|c|c|c|c|c|c|c|c|c|c|c|c|}
\hline F e $r \mathrm{me}$ & \multicolumn{2}{|c|}{$-1-$} & \multicolumn{2}{|c|}{$-2-$} & \multicolumn{2}{|c|}{$-3-$} & \multicolumn{2}{|c|}{$-4-$} & \multicolumn{2}{|c|}{$-5-$} & \multicolumn{2}{|c|}{$-6-$} & \multicolumn{2}{|c|}{$-7-$} & \multicolumn{2}{|c|}{$-8-$} \\
\hline $\mathrm{C} \circ \mathrm{m} \mathrm{m} \mathrm{n} e$ & \multicolumn{2}{|c|}{ Palmira } & \multicolumn{2}{|c|}{ Candelaria } & \multicolumn{2}{|c|}{ Candelaria } & \multicolumn{2}{|c|}{ Palmira } & \multicolumn{2}{|c|}{ Cartago } & \multicolumn{2}{|c|}{ Tulua } & \multicolumn{2}{|c|}{ Buga } & \multicolumn{2}{|c|}{ Buga } \\
\hline Annếe de rêfërence & 1967 & 1970 & 1969 & 1970 & 1968 & 1970 & 1969 & 1970 & 1969 & 1970 & 1967 & 1969 & 1969 & 1970 & 1968 & 1970 \\
\hline $\begin{array}{l}\text { Superficie agricole } \\
\text { utile (ha) }\end{array}$ & 15,4 & 16,0 & 21,848 & 21,848 & 37,12 & 38,0 & 110,0 & 83,2 & 54,4 & 54,4 & 94,4 & 104,4 & 50,1 & 50,1 & 140 & 140 \\
\hline $\begin{array}{l}\text { Superficie fourragère } \\
\text { pour l'exploitation } \\
\text { laitière (ha) }\end{array}$ & 15,4 & 15,36 & 21,848 & 21,848 & 37,12 & 38,0 & 110,0 & 83,2 & 54,4 & 54,4 & 28,8 & 30,7 & 36,3 & 21,7 & 140 & 77 \\
\hline $\begin{array}{l}\text { Vaches présentes } \\
\text { (moyenne annuelle) }\end{array}$ & 42,5 & 47,5 & 24,5 & 34,5 & 86,5 & 97 & 164 & 160,5 & 81 & 76 & 52 & 50,5 & 105,5 & 87 & 116,5 & 91,5 \\
\hline $\begin{array}{l}\text { UGB/ha de superficie } \\
\text { fourragère }\end{array}$ & 3,8 & 3,9 & 1,75 & 2,4 & 3,9 & 3,6 & 1,69 & 2,3 & 2,22 & 2,4 & 3,4 & 2,1 & 3,5 & 5,3 & 1,34 & 1,8 \\
\hline $\begin{array}{l}\mathrm{kg} \text { de lait/vaches } \\
\text { présentes/an }\end{array}$ & 1570 & 2528 & 3505 & 3381 & 2635 & 3471 & 2090 & 2207 & 2828 & 3095 & 1810 & 2516 & 2336 & 2902 & 1725 & 2240 \\
\hline $\begin{array}{l}\text { kg de lait produits } \\
\text { par an (total) }\end{array}$ & 66726 & 120088 & 85888 & 116635 & 227902 & 336690 & 342756 & 354291 & 228358 & 235702 & 60345 & 127059 & 241795 & 209028 & 201120 & 211753 \\
\hline $\begin{array}{l}\text { kg de lait produits } \\
\text { par an }\end{array}$ & 4333 & 7818 & 3931 & 5338 & 6140 & 8860 & 3116 & 4258 & 4197 & 4333 & 2095 & 4139 & 6661 & 9633 & 1437 & 2750 \\
\hline $\begin{array}{l}\text { Produit brut de I'ex- } \\
\text { ploitation laitière } \\
\text { par an (en pesos) }\end{array}$ & 116515 & 368128 & 220143 & 431810 & 373316 & 1210632 & 817335 & 1086402 & 602212 & 810451 & 864001 & 1425011 & 293974 & 535894 & 504567 & 495860 \\
\hline $\begin{array}{l}\text { Produit brut/an/ha } \\
\text { (en pesos) }\end{array}$ & 10813 & 23008 & 10076 & 19764 & 10057 & 31858 & 7430 & 13058 & $1108 \mathrm{~B}$ & 14898 & 9138 & 13650 & 8098 & 24696 & 3604 & 6440 \\
\hline $\begin{array}{l}\text { Rêsultat net de l'ex- } \\
\text { ploitation laitiëre } \\
\text { (1oyer inclus) par } \\
\text { an (en pesos) }\end{array}$ & 14026 & 108933 & 18404 & 63457 & -244693 & 373610 & 98582 & 233065 & -75947 & 115872 & 90981 & 626708 & -173153 & -34326 & -41572 & 52709 \\
\hline $\begin{array}{l}\text { Résultat net/ha/an } \\
\text { (en pesos) }\end{array}$ & 911 & 6808 & 842 & 2904 & -6592 & 9831 & 897 & 2801 & 1396 & 2130 & 965 & 6004 & -4770 & -1582 & -296 & 684 \\
\hline $\begin{array}{l}\text { Intërêt du capital } \\
\text { d'exploitation } \\
\text { (en } 7 \text { ) }\end{array}$ & 4,3 & 31,4 & 7,3 & 17,3 & $-35,8$ & 46,4 & 9,4 & 19,4 & $-10,5$ & 14,7 & 12,7 & 57,8 & $-29,7$ & $-4,3$ & $-4,5$ & 11,3 \\
\hline $\begin{array}{l}\text { Intërêt du capital } \\
\text { global investi } \\
\text { (en \%) }\end{array}$ & 5,8 & 20,6 & 6,0 & 13,2 & $-11,3$ & 29,2 & 7,6 & 13,6 & $-0,2$ & 5,2 & 8,7 & 19,2 & $-4,6$ & 0,16 & 5,5 & 8,6 \\
\hline
\end{tabular}


TABLEAU $N^{\circ}$ II

Evolution technique dans 20 p.100 des fermes laitières inscrites au programme depuis le début.

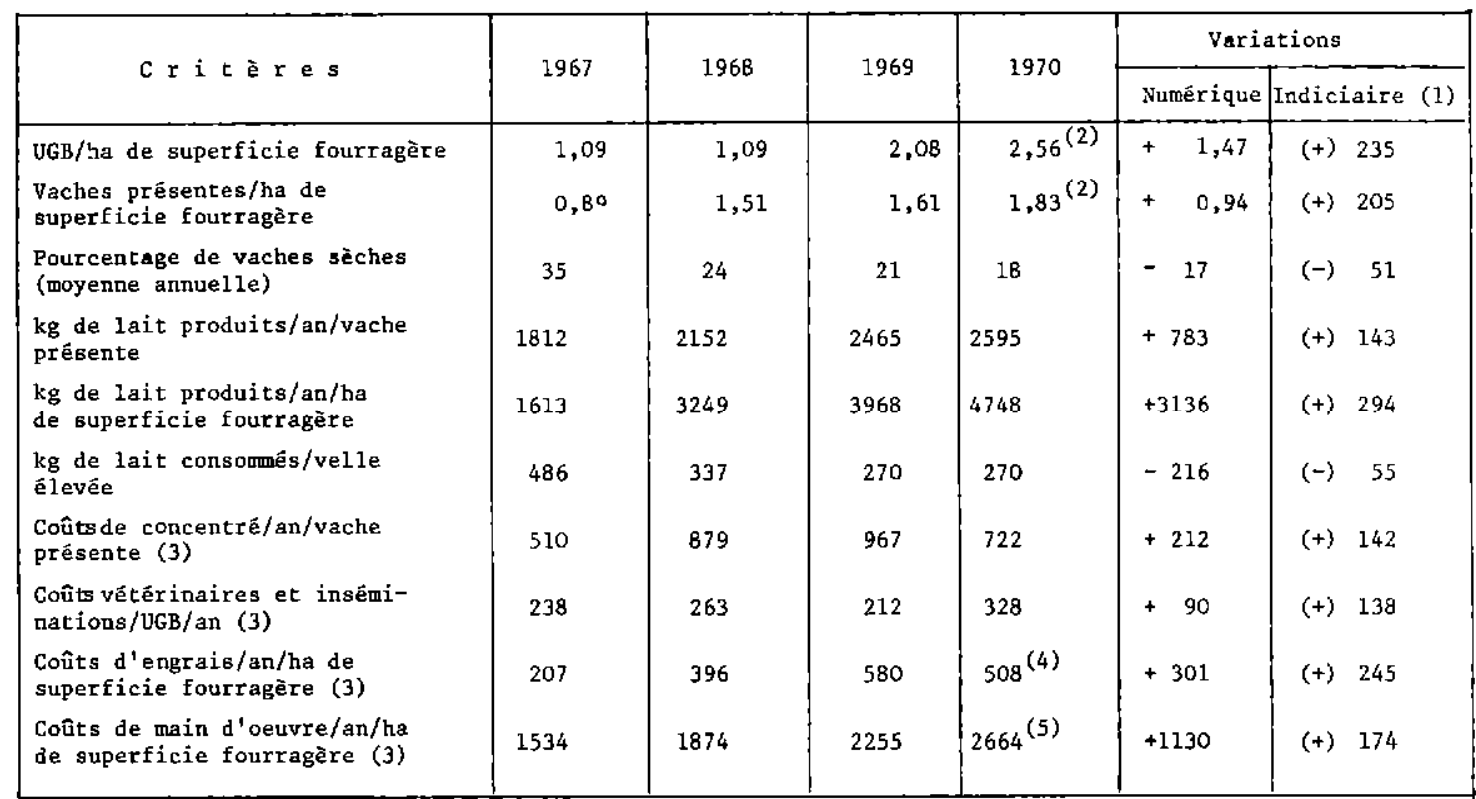

\section{Définitions de certains critères}

- Le produit brut comprend surtout la vente de lait. II comprend aussi le lait autoconsommé, le lait bu par les veaux ainsi que les ventes de bétail et les variations d'inventaire (changements de catégories des animaux).

- Le résultat net envisagé est la différence annuelle entre le produit brut de l'exploitation et les coûts globaux (loyer de la terre inclus).

- L'intérêt du capital d'exploitation est le quotient $\times 100$ de ce résultat net par le capital d'exploitation (matériel et surtout animaux).

- L'intérêt du capital global investi est le quotient $\times 100$ du résultat net de la ferme en propriété (sans le loyer de la terre) par le capital agricole global (capital foncier + capital d'exploitation).

\section{Observations}

Entre 1967 et 1970 la SAU de certaines exploitations s'est modifiée (certaines se sont agrandies, d'autres ont diminué de taille). D'autres fermes, dans le processus d'intensification, ont augmenté leurs cultures et réduit leur superficie fourragère (cas des fermes 7 et 8 ), ce qui leur a permis d'effectuer une sévère sélection des vaches.
Noter la grande variation des charges à l'ha.

Noter les variations souvent spectaculaires du produit brut, dues d'une part à l'augmentation combinée de la charge et de la production individuelle et aussi à l'augmentation du prix du lait.

La ferme 7, malgré sa charge très élevée, continue à avoir en 1970 un intérêt du capital d'exploitation négatif: ses coûts en maind'œuvre et en aliments concentrés étant trop élevés.

\section{Observations: sur tableau II}

1. En affectant de l'indice 100 les chiffres de l'année 1967.

2. Les chiffres obtenus en 1970 sont encore bas par rapport au potentiel de la région et sont encore bien inférieurs aux objectifs fixés qui sont pour les bonnes terres :

- 4 UGB dont 2,7 vaches/ha de surface fourragère;

- plus de $8.000 \mathrm{~kg}$ de lait/ha/an; chiffres qui ont été obtenus et même dépassés dans certaines exploitations.

3. Ces coûts comparés sont exprimés en pesos colombiens (actuellement 1 US $\$=$ 20 pesos au cours officiel).

4. L'intensification fourragère implique une augmentation du coût des engrais (surtout azo- 
tés). Les coûts enregistrés en 1970 sont encore trop faibles en général et sont le reflet des progrès qu'il y a encore à faire dans l'augmentation de la charge et de la production de lait par ha.

5. L'intensification laitière (augmentation du nombre d'animaux) se traduit par la création d'emplois et une augmentation des coûts de main-d'œuvre (relativement encore très bon marché dans la région). Toutefois, les coûts de main-d'œuvre doivent être limités le plus possible pour ne pas grever inutilement le coût de production du lait.

\subsection{Conclusion}

Ces résultats réels qui ont pu être mesurés facilement par la méthode de travail employée prouvent, plus que tout commentaire, la valeur du programme développé, le bien-fondé et l'utilité de la Mission technique française qui l'a conçu et aidé dans ses premiers pas.

\section{SUMMARY}

Spectacular increase of milk production in the

Rio Cauca Valley. Colombia

Methods and results

In this very fertile valley (altitude 900 to 1000 meters) with a hot and humid tropical climate (latitude $4^{\prime \prime}$ north), a group of French and Colombian experts investigated how to rationally use conventional methods dealing with grassland and livestock that would be suited to local conditions as well as how to improve the quality of the dairy farmer's management efforts and the extent of his technical know-how.

This article analyzes the important results obtained in eight pilot farms with a total of 650 cows. Within two years, the annual production of milk rose from 4000 kilograms per hectare to 5900 kilograms per hectare, or in other words, increased the production by about 47 p. 100 and farm profitability soared.

The program's objective is to assist 200 farms covering an area of 25000 hectares and producing on the better dairy farms, 8000 kilograms of milk per hectare each year (a figure already attained on some of the farms).

The fact that there is a shortage of milk in the country is what makes this program worthwhile from an economic and social stanpoint.

\section{RESUMEN}

Mejoramientos espectaculares de la produccion de la leche en el Valle del Cauca (Colombia)

En este valle muy fértil, un grupo de profesionales colombianos y franceses, enfocándose a la vez sobre los pastos, el ganado y el hombre, buscó el uso racional de métodos clásicos adaptados al medio y sobretodo la modificación de la mentalidad y de las cualidades administrativas y técnicas de Ios propietarios de fincas.

Este artículo analizá los resultados interesantes obtenidos en 8 fincas pilotos que agrupan un total de 650 vacas. En este grupo de fincas la producción lechera subió en 2 años de $4000 \mathrm{~kg}$ à $5900 \mathrm{~kg}$ de leche/ha/año, o sea un aumento de producción de 47 p. 100 más o menos. Se logró sobre todo un aumento espectacular de la rentabilidad de estas explotaciones.

La meta de la producción en las buenas fincas jecheras es $8000 \mathrm{~kg}$ de leche/ha/año (producción ya alcanzada en unas explotaciones).

El país presenta escasez de leche, lo cual justifica el interés económico y social de este tipo de programa. 